\title{
Esquizofrenia: O Que o Médico Não Psiquiatra Precisa de Saber
}

\section{Schizophrenia: What Non-Psychiatrist Physicians Need to Know}

\author{
Tiago QUEIRÓS $\triangle^{1}$, Filipa COELHO ${ }^{1}$, Ludgero LINHARES ${ }^{1}$, Diogo TELLES-CORREIA ${ }^{1,2}$
}

Acta Med Port 2019 Jan;32(1):70-77 - https://doi.org/10.20344/amp.10768

\begin{abstract}
RESUMO
A esquizofrenia é uma doença mental grave e incapacitante que afeta todas as classes sociais e raças, em todas as partes do mundo sendo mais frequente no sexo masculino. Manifesta-se habitualmente na parte final da adolescência ou início da vida adulta e é importante a sua deteção precoce por todos os clínicos para correto encaminhamento para consulta especializada de psiquiatria. Pretende-se com este artigo atualizar conhecimentos em relação ao diagnóstico, tratamento e prognóstico da esquizofrenia e enfatizando os sinais de alerta para uma referenciação atempada a consulta de psiquiatria. Foi efetuada uma pesquisa bibliográfica através de artigos disponíveis em bases de dados de artigos científicos e também em livros científicos e técnicos especializados na área da esquizofrenia. A apresentação clínica desta doença é heterogénea e complexa, com uma evolução típica normalmente pautada por vários episódios de descompensação aguda com necessidade de internamento. O diagnóstico de esquizofrenia assenta em alguns sintomas-chave, sendo que os vários critérios de diagnóstico internacionais variam entre eles relativamente à janela temporal com sintomatologia produtiva necessária para efetuar um diagnóstico. O prognóstico é muito variável, nem sempre cursa de forma deteriorante e é tanto melhor quando mais precoce for o início do tratamento. $O$ tratamento exige uma abordagem multidisciplinar e assenta primariamente em fármacos antipsicóticos. Esta medicação apesar de muito eficaz para a sintomatologia típica da doença acarreta efeitos adversos cujas consequências médicas são importantes na prática clínica de todos os médicos de outras especialidades.
\end{abstract}

Palavras-chave: Antipsicóticos; Esquizofrenia/diagnóstico; Esquizofrenia/tratamento; Perturbações Psicóticas

\section{ABSTRACT}

Schizophrenia is a disabling and severe mental illness that affects all social classes and racial and ethnic groups, spreading across every part of the world. It's more frequent in males and it usually manifests itself in late adolescence or early adulthood and its early detection by all clinicians is important so that there is a proper referral to specialized psychiatric care. This article intends to update the knowledge regarding the diagnosis, treatment and prognosis of schizophrenia, with an emphasis on the warning signs for a timely referral to psychiatric evaluation. We conducted a literature search across through articles available in databases of scientific articles but also in scientific and technical books specialized in the field of schizophrenia. The clinical presentation of this illness is heterogeneous and complex, with a typical evolution based on several episodes of acute decompensation requiring hospitalization. The diagnosis of schizophrenia relies on some key symptoms, and the various international diagnostic criteria vary in relation to the temporal window with productive symptomatology required to establish a diagnosis. The prognosis is variable, not always deteriorating and is all the better when the treatment is started as early as possible. Treatment requires a multidisciplinary approach and is based primarily on antipsychotic drugs. This medication although very effective for the typical symptoms of this illness, entails some adverse effects with medical consequences that are important in the clinical practice of all doctors of other specialties.

Keywords: Antipsychotic Agents; Psychotic Disorders; Schizophrenia/diagnosis; Schizophrenia/therapy

\section{INTRODUÇÃo}

A esquizofrenia é uma das doenças mentais graves mais frequentes e tem sido identificada como uma prioridade em termos de políticas de saúde devido ao défice de funcionamento inerente e à mortalidade precoce. ${ }^{1}$

Esta entidade nosológica engloba, provavelmente, um grupo de doenças de etiologias diversas, cuja apresentação clínica, resposta ao tratamento e evolução podem variar. ${ }^{2}$ Identificada em todas as partes do mundo, em todas as classes sociais e etnias, as taxas de incidência diferem entre 7,7 e 43,0 por 100000 habitantes, sendo esta maior em meios urbanos e em classes sociais mais desfavorecidas. A incidência parece, ainda, ser significativamente maior em indivíduos do sexo masculino, com um rácio de 1,4 em relação ao sexo feminino. ${ }^{3} \mathrm{Na}$ maioria dos estudos, a prevalência ronda os 2,1-7,0 em cada 1000 habitantes. ${ }^{4}$
Tanto os doentes e suas famílias, como a sociedade sofrem um impacto económico importante. As hospitalizações representam o custo direto mais substancial da esquizofrenia, do ponto de vista dos cuidados de saúde. ${ }^{5}$

As primeiras manifestações da doença surgem, habitualmente, na parte final da adolescência ou no início da vida adulta. Em doentes do sexo masculino ocorrem geralmente entre os 15 e os 25 anos e, no caso do sexo feminino, observa-se uma distribuição etária bimodal, com um primeiro pico entre os 25 e 30 anos e um segundo pico mais tarde na idade adulta (entre $3 \%$ a $10 \%$ das mulheres têm o início da doença após os 40 anos de idade). ${ }^{6}$

A taxa de mortalidade por acidente e doença natural é mais elevada nesta população. Estudos recentes mostram que estes doentes morrem, em média, 15 a 20 anos mais

1. Serviço de Psiquiatria. Hospital de Santa Maria. Centro Hospitalar Lisboa Norte. Lisboa. Portugal.

2. Departamento de Psiquiatria. Faculdade de Medicina. Universidade de Lisboa. Lisboa. Portugal.

$\triangle$ Autor correspondente: Tiago Queirós. tiagopintoqueiros@gmail.com

Recebido: 09 de maio de 2018 - Aceite: 29 de outubro de 2018 | Copyright @ Ordem dos Médicos 2019 
cedo do que a população geral, com maior prevalência de doença cardiovascular e neoplásica e diabetes mellitus. Os efeitos adversos da medicação antipsicótica, a escassez de atividade física, a dieta e os hábitos tabágicos importantes são fatores que contribuem para o aparecimento de comorbilidades. ${ }^{7}$ Condicionantes como o estigma associado às doenças psiquiátricas, o desafio clínico que pode moldar o seu diagnóstico e o tratamento das patologias médicas têm sido considerados relevantes. ${ }^{2}$

Dada a importância da sua deteção e tratamento precoces torna-se crucial a intervenção dos médicos de outras especialidades, com particular relevo os médicos de família, na deteção e encaminhamento a consulta de psiquiatria. Por outro lado, o seu papel é também fundamental na promoção da qualidade de vida, no apoio familiar e social, e no diagnóstico e acompanhamento de possíveis comorbilidades, algumas associadas ao próprio tratamento psicofarmacológico da doença. ${ }^{1}$

\section{QUAL A ETIOPATOGENIA DA ESQUIZOFRENIA?}

Face às investigações das últimas décadas, considera-se, atualmente, uma etiologia multifatorial, com a contribuição de fatores psicossociais e biológicos. Fatores hereditários parecem contribuir de forma importante no aparecimento da doença, existindo um grande número de genes envolvidos. Quanto à transmissão, a investigação avança como mais provável um modelo poligénico, com alelos comuns e raros, cada um com efeito muito pequeno no risco global. Estes podem interferir na migração e plasticidade neuronal, na sinaptogénese e na atividade da dopamina, do glutamato e da acetilcolina. ${ }^{4}$

Vários fatores ambientais têm sido associados à doença, nomeadamente complicações obstétricas e perinatais, infeções e malnutrição materna, nascimentos nos meses de inverno, urbanidade, migração e consumo de substâncias. Este último, em particular o consumo de canabinóides, pode constituir um fator precipitante em indivíduos predispostos ou surgir como um fator secundário ao início dos sintomas, para além de conferir pior prognóstico. ${ }^{4}$

Várias hipóteses associadas a neurotransmissores têm sido propostas, implicando o papel da dopamina, serotonina, glutamato e ácido gama-aminobutírico. Recentemente, observou-se um aumento na concentração sérica de várias citocinas pró-inflamatórias associado ao stress oxidativo. A hipótese do neurodesenvolvimento contempla a possibilidade da origem da doença numa perturbação do desenvolvimento ou maturação do cérebro no período perinatal. ${ }^{6}$

A partir da década de 40, surgiram teorias psicológicas tentando explicar a esquizofrenia a partir de relacionamentos familiares patológicos e padrões de comunicação interpessoal aberrantes, como a ideia da 'mãe esquizofrenizante' que assume padrões de comportamento de hostilidade e rejeição e a teoria de double bind, que pressupõe uma sequência de experiências insolúveis, e que é responsável pela produção de conflitos internos de classificação lógica. ${ }^{8,9}$ Têm sido feitos esforços no sentido de integrar os vários modelos etiopatogénicos.

\section{DE QUE FORMA SE APRESENTA A ESQUIZOFRENIA?}

O quadro clínico é bastante heterogéneo, complexo e nem sempre facilmente percetível - nenhum sinal ou sintoma é por si só patognomónico e estes variam ao longo do curso da doença. É importante salientar que todos os sintomas e sinais da esquizofrenia são também encontrados frequentemente noutras patologias psiquiátricas e neurológicas. $2,4,6,10$

De forma típica, no decurso da fase pré-mórbida variável de um doente com esquizofrenia podem ser encontrados traços de personalidade esquizoide ou esquizotípica (calados, introvertidos, pouco comunicativos, passivos). Na infância e na adolescência podem ter poucos amigos e excluir-se de atividades sociais ou relações afetivas. Pelo contrário preferem atividades mais solitárias como ver televisão, ouvir música ou jogar jogos de computador. Nesta fase o doente pode desenvolver um interesse em ideias abstratas, por filosofia, oculto pelo oculto, por religião ou até desenvolver sintomatologia obsessiva. Todos estes fatores deverão ser avaliados em consultas relativas ao Programa Nacional de Intervenção em Saúde Infantil e Juvenil, pelo médico de medicina geral e familiar. 2,4,6,11 Classicamente podemos dividir os sintomas da esquizofrenia em sintomas positivos, negativos e em outros sintomas (cognitivos e afetivos).

\section{QUAIS SÃO OS SINTOMAS POSITIVOS E NEGATIVOS?}

Os sintomas positivos (também designados por sintomas produtivos) são os sintomas presentes de forma mais visível nas fases de descompensação aguda da doença e incluem classicamente os delírios e as alucinações. São também incluídas neste conjunto outras alterações do pensamento, discurso ou comportamento..$^{2,4,6,12}$ Genericamente os delírios são definidos como uma crença individual falsa que é irredutível perante argumentação lógica, não partiIhada por um grupo de pessoas da mesma cultura e de conteúdo implausível. Este último fator é controverso (visto poderem existir delírios próximos da plausibilidade, como na perturbação delirante) e é substituído por alguns autores por incompreensibilidade psicológica. ${ }^{13}$ Os delírios mais comuns nos doentes com esquizofrenia são o delírio persecutório (o doente tem ideias de prejuízo contra si, sente-se ameaçado, perseguido, espiado ou vítima de uma conspiração) e o delírio de autorrelacionação (o doente acredita que as experiências são dirigidas a si, que falam sobre si, frequentemente em tom depreciativo ou que por exemplo mensagens em programas de televisão são para si).4,6,14,15 Uma alucinação frequentemente é definida por "uma perceção sem objeto" e será melhor definida como uma falsa perceção que ocorre ao mesmo tempo que as perceções reais, mantendo a consistência sensorial, localizando-se no espaço exterior e objetivo, tendo todo o detalhe e clareza, sendo constante ao longo do tempo e não sendo alterada pela vontade. ${ }^{13}$ As alucinações são muito frequentes na esquizofrenia e podem ser de qualquer modalidade sensorial. ${ }^{6}$ As mais comuns são as auditivo-verbais e tomam a forma de vozes que são frequentemente desagradáveis, críticas, ameaçadoras, obscenas ou insultuosas. Podem ser ape- 
nas uma voz ou mais, a conversar entre si, a comentar o comportamento do doente e o que se passa à sua volta, ou com carácter de comando, dando ordens. A presença de alucinações visuais, olfativas ou gustativas é incomum, devendo alertar o clínico para a possibilidade de um quadro orgânico como causa subjacente da síndrome. .,4,6,14-16 $^{2}$

Outras alterações do pensamento são também comuns na esquizofrenia. Podem ser estabelecidas associações sem qualquer nexo ou uma paragem súbita na linha de pensamento (bloqueio do pensamento) criando uma verdadeira dificuldade em manter uma conversa, que pode ser percecionada numa consulta médica. Os doentes podem relatar experiências de alienação do seu próprio pensamento por agentes externos sendo sintomas-chave no diagnóstico de esquizofrenia (nomeadamente controlo, inserção, roubo ou difusão do pensamento). As alterações do pensamento podem ser um sinal de alerta para o clínico e podem ser facilmente detetadas pela sua estranheza. $2,4,6,14,15$

O discurso pode tornar-se estranho, incompreensível e sem lógica. O doente pode exprimir-se de forma desorganizada através de uma série de palavras isoladas sem qualquer sentido (salada de palavras), de novas palavras inventadas pelo doente (neologismos) ou através de respostas ao lado. ${ }^{2,4,6,14,15}$

Existem também frequentemente alterações no comportamento manifestando-se através de auto ou hétero-agressividade, agitação e inquietude motoras, atitudes bizarras ou isolamento. Contrariamente, há casos em que a atividade motora é praticamente nula (estupor catatónico). O doente também pode apresentar uma postura ou atitude contrárias às solicitadas pelo interlocutor (negativismo) ou repetir movimentos imitando o interlocutor (ecopraxia). ${ }^{2,6}$

Os doentes com esquizofrenia de longa evolução podem perder a noção de regras sociais e de higiene. Podem ser encontrados a falar sozinhos (solilóquios), a utilizar uma linguagem obscena (coprolalia) ou com risos imotivados (o doente ri-se daquilo que as vozes lhe dizem). ${ }^{2,6}$

Classicamente os sintomas negativos são um conjunto de sintomas que representam a perda ou diminuição das funções normais. Este grupo de sintomas acompanha a evolução da doença e refletem um estado deficitário ao nível da motivação, das emoções, do discurso, do pensamento e das relações interpessoais. ${ }^{4,17-19}$

Os sintomas negativos podem ser primários (resultantes da própria evolução da esquizofrenia) ou secundários (aos próprios sintomas positivos, à desorganização, à depressão, ao isolamento social ou aos efeitos da medicação antipsicótica). ${ }^{2,4,6,17} \mathrm{O}$ Manual de Diagnóstico e Estatística das Perturbações Mentais, $5^{\text {a }}$ edição (DSM-5) definiu como sintomas negativos os seguintes: aplanamento e embotamento afetivos (redução da amplitude e intensidade da expressão emocional), alogia (pobreza no discurso com respostas lacónicas, sem conteúdo), avolição (redução da motivação, falta de vontade e de iniciativa), anedonia (perda da habilidade em sentir prazer em atividades ou relacionamentos interpessoais) e isolamento social. A Classificação Estatística Internacional de Doenças e Problemas
Relacionados com a Saúde, $10^{a}$ edição (CID-10) define os sintomas negativos como marcada apatia, escassez de discurso e aplanamento ou incongruência das respostas emocionais. ${ }^{4,17-19}$

A gravidade dos sintomas negativos vai influenciar o funcionamento global do indivíduo, a autonomia, o prognóstico e a evolução da doença. Compreender e tratar melhor estes sintomas irá aumentar a qualidade de vida e participação na sociedade destes indivíduos. 2,4,6,17

\section{QUE OUTROS SINTOMAS PODEM EXISTIR NA ESQUI- ZOFRENIA?}

Existem outros sintomas que não fazem parte dos critérios formais do diagnóstico de esquizofrenia e que vão além dos sintomas positivos e negativos. Alterações cognitivas encontram-se habitualmente presentes na altura da manifestação da doença (ou mesmo numa fase prodrómica) e parecem ser os principais determinantes do funcionamento e reabilitação psicossociais. São encontrados défices mais marcados a nível da memória verbal, da vigilância e atenção, da memória de trabalho, do quociente intelectual, da linguagem e das funções executivas. As alterações cognitivas são relativamente independentes dos restantes sintomas da doença, sendo mais comuns nos doentes com predomínio de sintomatologia negativa. ${ }^{4,6,20}$ (Tabela 1)

Sintomatologia afetiva também é comum nos doentes com esquizofrenia ao longo da sua vida, nomeadamente sintomatologia depressiva (em cerca de $40 \%$ dos casos). ${ }^{6,21,22}$

\section{COMO É FEITO O DIAGNÓSTICO DE ESQUIZOFRENIA?}

O diagnóstico de esquizofrenia é, muitas vezes, difícil, já que para além de ser longitudinal assenta principalmente na história clínica e na observação psicopatológica. Atualmente, não dispomos de exames laboratoriais ou imagiológicos que possibilitem per se o diagnóstico. Contudo, têm sido encontradas algumas alterações neuroanatómicas e certos biomarcadores na esquizofrenia, embora não sejam específicos da doença e a sua validade seja controversa. $2,4,6,23,24$

Tabela 1 - Sintomas-chave no diagnóstico da esquizofrenia

\section{Sintomas-chave da esquizofrenia}

\section{Sintomas Positivos:}

- Delírios (principalmente persecutório e de autorrelacionação);

- Alucinações (principalmente auditivo-verbais);

- Alterações do curso, posse e forma do pensamento;

- Alterações comportamentais.

\section{Sintomas Negativos:}

- Aplanamento e embotamento afetivos;

- Alogia;

- Avolição;

- Anedonia;

- Apatia;

- Isolamento social.

Outros Sintomas:

- Sintomas cognitivos;

- Sintomas afetivos. 
Os dois sistemas de classificação internacional mais utilizados atualmente como apoio ao diagnóstico de esquizofrenia são o CID-10 e o DSM-5. Apesar de coincidentes em alguns aspetos, os critérios de ambos apresentam importantes diferenças que são resumidas na Tabela 2. Torna-se assim importante a adequada referenciação ao médico psiquiatra na presença de sintomatologia suspeita já que este complexo diagnóstico acarreta consigo um estigma social. A interpretação incorreta e precoce de vários sintomas psiquiátricos como esquizofrenia poderá suscitar angústia e revolta contra os técnicos de saúde, levando a um abandono do plano terapêutico. 4,18,19

Dos critérios de diagnóstico de esquizofrenia segundo o DSM-5 foram eliminados os subtipos de esquizofrenia anteriormente presentes na $4^{a}$ edição do manual (paranóide, hebefrénica, catatónica, indiferenciada, residual e simples). Estes subtipos têm limitada estabilidade de diagnóstico, baixa confiabilidade, fraca validade e pouca utilidade clínica. No entanto, a CID-10 mantém a divisão do diagnóstico em subtipos. ${ }^{18,19,25,26}$

Por fim, é importante referir que relativamente ao diagnóstico diferencial devemos considerar três grandes grupos: 1) perturbações do 'espectro da esquizofrenia' tais como a perturbação esquizotípica, perturbação delirante, perturbação esquizoafetiva, perturbação psicótica aguda e transitória; 2) perturbações nosograficamente distintas da esquizofrenia tais como quadros afetivos que cursam com sintomas psicóticos, perturbações da personalidade, etc; 3) quadros orgânicos com manifestações psicóticas. ${ }^{2,4}$ Deste último grupo salientam-se as lesões ocupantes de espaço, epilepsia, encefalites auto-imunes, endocrinopatias ou um quadro infecioso. ${ }^{4}$

Relativamente à utilização de anticorpos onconeuronais em doentes com sintomas psiquiátricos, a prevalência

Tabela 2 - Critérios de diagnóstico de esquizofrenia, apresentados esquematicamente, segundo o CID-10† e DSM- $5^{\star 4,19}$

\begin{tabular}{lll} 
& CID $-10 \dagger$ & DSM $-5 *$ \\
Categoria & F20 & 295.90 \\
\hline
\end{tabular}

Sintomas Característicos:

- Um ou mais dos seguintes sintomas:

$\mathrm{Ou}$

- Dois ou mais dos seguintes sintomas:
Curso
- Um mês.

- Eco, inserção, roubo ou difusão do pensamento; - Delírios de controlo, influência ou passividade; - Delírios persistentes de outros tipos;

- Alucinações auditivo-verbais sob a forma de vozes que podem comentar ou discutir os comportamentos do doente ou provenientes de qualquer parte do corpo;

\begin{abstract}
- Alucinações persistentes de qualquer modalidade; em incoerência no discurso ou neologismos;

- Comportamento catatónico tal como excitação, posturas inadequadas ou flexibilidade cérea, negativismo, mutismo e estupor;

- Sintomas negativos tais como apatia marcada, escassez de discurso e embotamento ou incongruência de respostas emocionais, com compromisso social (deve estar claro que estes não são devidos a depressão ou medicação neuroléptica); - Alterações significativas na sua personalidade (manifestando-se com falta de interesse, falta de propósito, ociosidade e compromisso social).
\end{abstract}

- Delírios;

- Alucinações;

- Discurso desorganizado (por exemplo descarrilamento ou incoerência frequentes);

- Comportamento grosseiramente desorganizado ou catatónico;

- Sintomas negativos (por exemplo embotamento afetivo, alogia, avolição).

Nota: Pelo menos um dos três primeiros deve estar presente.

- Seis meses (incluindo um mês em fase aguda e períodos prodrómicos ou com sintomatologia residual);

- Com impacto no funcionamento global;

- Perturbação esquizoafetiva;

- Perturbação depressiva ou bipolar com sintomas psicóticos;

- Perturbação não atribuível a efeitos fisiológicos de substâncias ou a condição médica.

† Classificação Estatística Internacional de Doenças e Problemas Relacionados com a Saúde, $10^{\mathrm{a}}$ edição. * Manual de Diagnóstico e Estatística das Perturbações Mentais, $5^{\mathrm{a}}$ edição. 
destes em doentes com patologia psiquiátrica é relativamente baixa; no entanto, esta é uma área em expansão e que poderá alterar a maneira como abordamos estes doentes. ${ }^{27}$

\section{COMO É A EVOLUÇÃO E O PROGNÓSTICO?}

Caracteristicamente, os sintomas pré-mórbidos começam na adolescência e são reconhecidos de forma retrospetiva. Mudanças no ambiente externo (entrar na faculdade, mudar de cidade, uso de substâncias, morte de familiar, etc.) podem precipitar estes sintomas que podem persistir durante anos antes da instalação da sintomatologia psicótica. Desta forma é importante que o médico de família esteja alerta para estes sintomas, acompanhe a evolução e eventualmente sinalize estes jovens para consulta de psiquiatria. $2,4,6,28$

A evolução clássica da esquizofrenia é pautada por repetidas exacerbações da doença (geralmente por sintomas positivos) com remissões e recaídas com vários internamentos. A maioria dos doentes responde favoravelmente ao tratamento com antipsicóticos, contudo aproximadamente $80 \%$ recaem nos primeiros cinco anos após o episódio inaugural, o que é parcialmente explicado pela descontinuação da terapêutica. Cada recaída da doença representa uma deterioração no nível funcional prévio do doente e esta é a característica que separa a esquizofrenia das perturbações do humor (ex: perturbação afetiva bipolar). Os sintomas positivos tendem a ser menos intensos com o tempo e passa a haver um predomínio da sintomatologia negativa e cognitiva, causando um grande impacto na vida pessoal, social e laboral do indivíduo. . $^{2,4,6,28}$

A visão clínica e social tradicional da esquizofrenia é de uma doença deteriorante e debilitante com mau prognóstico. Vários estudos mostraram que no período de 5 a 10 anos após o primeiro internamento em psiquiatria, apenas $10 \%-20 \%$ dos doentes mostraram ter bom prognóstico. Mais de $50 \%$ apresentaram mau prognóstico com internamentos repetidos, exacerbações dos sintomas e tentativas de suicídio. Estima-se que apenas $20 \%$ a $30 \%$ dos doentes consigam viver uma vida normal. 2,4,6,29

Desta forma, o prognóstico da esquizofrenia é caracterizado por uma grande heterogeneidade e a doença nem sempre cursa de forma deteriorante já que alguns fatores foram associados com bom prognóstico (Tabela 3). É importante para os clínicos a compreensão destes dados e a sua transmissão de forma empática ao doente e à família, pela importância em derrubar estereótipos, já que existe, à partida, uma errada suposição de que será sempre de mau prognóstico. 2,4,6,29

\section{COMO SE TRATA?}

$O$ tratamento da esquizofrenia assenta numa abordagem multidisciplinar e visão longitudinal, considerando as diferentes fases da doença. Devem ser considerados os domínios biológico, psicológico e social do indivíduo, na procura do seu melhor funcionamento e na prevenção do declínio cognitivo. $4,6,31,32$

Tabela 3 - Fatores de bom e mau prognóstico na esquizofrenia ${ }^{2,4,30}$

\section{Fatores de prognóstico na esquizofrenia}

Fatores de bom prognóstico:

- Sexo feminino;

- Casado;

- Idade de início tardia;

- Fatores precipitantes óbvios;

- Funcionamento pré-mórbido (social, sexual, laboral) ajustado;

- Boa rede de suporte familiar e social;

- Família com baixo nível de emotividade expressa;

- Início agudo;

- Tratamento precoce;

- Episódio breve;

- Reduzido número de recaídas;

- História familiar de perturbações do humor;

- Predomínio de sintomas positivos;

- Predomínio de sintomas afetivos (especialmente depressivos);

- Boa adesão à terapêutica;

- Boa resposta à terapêutica;

- Sem sinais e sintomas neurológicos;

- Sem história de trauma perinatal;

- Sem consumo de substâncias.

\section{Fatores de mau prognóstico:}

- Sexo masculino;

- Solteiro, divorciado ou viúvo;

- Idade de início precoce;

- Sem fatores precipitantes óbvios;

- Funcionamento pré-mórbido (social, sexual, laboral) pobre;

- Fraca rede de suporte familiar e social;

- Família com alto nível de emotividade expressa;

- Início insidioso;

- Tratamento tardio;

- Episódio de longa duração;

- Elevado número de recaídas;

- História familiar de esquizofrenia;

- Predomínio de sintomas negativos;

- Má adesão à terapêutica;

- Má resposta à terapêutica;

- Com sinais e sintomas neurológicos;

- Com história de trauma perinatal;

- Com consumo de substâncias.

O tratamento psicofarmacológico, mais concretamente a medicação antipsicótica, revolucionou os cuidados prestados a pessoas com esquizofrenia e continuam a ser a abordagem de primeira linha nesta doença. Não obstante, estes medicamentos acarretam vários efeitos adversos indesejados expectáveis que podem limitar a adesão à terapêutica, tornando-se vital conhecer os grupos de antipsicóticos existentes. De uma forma geral, os fármacos antipsicóticos podem ser divididos em dois grupos distintos, os de primeira geração (típicos) e os de segunda geração (atípicos), tal como listado na Tabela 4.,4,631,32

\section{COMO ATUAM OS ANTIPSICÓTICOS E QUAIS OS SIN- TOMAS ADVERSOS A TER EM ATENÇÃO?}

A característica universal dos antipsicóticos, tal como o próprio nome indica, é o tratamento da psicose (via mesolímbica), atuando por antagonismo ou agonismo parcial dos recetores da dopamina, sobretudo dos recetores do subtipo D2. No entanto, a variedade de afinidade para outros 
recetores (serotoninérgicos, colinérgicos $\mathrm{M} 1$, $\alpha 1$ adrenérgicos, histamina H1) confere a cada antipsicótico uma ação singular que deve ser considerada na prescrição. $4,6,31,32,34$

Os antipsicóticos de primeira geração (APG) atuam, sobretudo no bloqueio dos recetores D2 que, para além do efeito antipsicótico pretendido (obtido sobretudo pela sua ação na via dopaminérgica mesolímbica). Este mecanismo pode levar ao surgimento de sintomas extrapiramidais (acatísia, distonia aguda, parkinsonismo, discinesia tardia) e hiperprolactinemia que por vezes traduz-se clinicamente em amenorreia, galactorreia e disfunção sexual. 4,6,31,32,34

Os antipsicóticos de segunda geração (ASG), para além do bloqueio de recetores $\mathrm{D} 2$, bloqueiam também os recetores serotoninérgicos $5-\mathrm{HT} 2 \mathrm{~A}$. A estimulação destes recetores inibe a libertação de dopamina, logo o bloqueio dos mesmos conduzirá inversamente a aumento da libertação de dopamina. Este aumento de dopamina terá uma funcionalidade particular na via mesocortical ao levar uma melhoria dos sintomas negativos, o que corresponde a uma vantagem dos ASG face aos APG. ${ }^{4,6,31,32,34}$
Dependendo do antipsicótico, pode existir ainda bloqueio de outros recetores. Este bloqueio será responsável por outros efeitos secundários podendo, no entanto ter alguma utilidade clínica como o caso da sedação (utilizados em caso de agitação psicomotora). O bloqueio dos recetores $\alpha 1$ adrenérgicos pode estar associado a hipotensão postural, tonturas e taquicardia e o bloqueio dos recetores muscarínicos M1 associado a visão turva, xerostomia, obstipação, retenção urinária, agravamento de glaucoma de ângulo fechado e défices cognitivos. Os recetores $\mathrm{H} 1$ quando bloqueados conduzem a sedação e aumento do apetite. Outros efeitos secundários devem ser considerados como alterações eletrocardiográficas (prolongamento do intervalo QT) e alterações metabólicas como aumento de peso, dislipidemia e desenvolvimento de resistência à insulina, aumentando o risco de síndrome metabólica. ${ }^{4,6,31,32,34}$

De modo geral, considerando a dicotomia entre APG e ASG, podemos resumir que, no que concerne a efeitos adversos, os primeiros estão mais associados a sintomas extrapiramidais e os segundos a efeitos metabólicos.

Tabela 4 - Classificação dos antipsicóticos para o tratamento da esquizofrenia, separados por primeira e segunda geração 2,30,33

\begin{tabular}{ll}
\hline Antipsicóticos de primeira geração & Antipsicóticos de segunda geração \\
\hline - Cloropromazina; & - Amilsuprida; \\
- Flufenazina; & - Aripiprazol; \\
- Flupentixol; & - Asenapina; \\
- Haloperidol; & - Lusapina; \\
- Levomepromazina; & - Olanzapina; \\
- Pimozida; & - Paliperidona; \\
- Sulpirida; & - Quetiapina; \\
- Zuclopentixol. & - Risperidona; \\
& - Ziprasidona; \\
& - Zotepina. \\
\hline
\end{tabular}

Tabela 5 - Resumo dos principais efeitos adversos da terapêutica antipsicótica

Principais efeitos adversos da terapêutica antipsicótica

Extrapiramidais (mais associados aos APG †):

- Acatísia;

- Distonia aguda;

- Parkinsonismo;

- Discinesia tardia.

Metabólicos (mais associados aos ASG *):

- Aumento do apetite;

- Aumento de peso;

- Dislipidemia;

- Resistência à insulina;

- Elevado risco de desenvolver síndrome metabólica.

Outros:

- Hiperprolactinemia (causando amenorreia, galactorreia e disfunção sexual);

- Cardiovasculares (hipotensão postural, taquicardia e ECG com prolongamento do intervalo QT);

- Sedação;

- Tonturas;

- Visão turva;

- Xerostomia;

- Obstipação;

- Retenção urinária;

- Agravamento do glaucoma de ângulo fechado;

- Défices cognitivos.

† APG: antipsicóticos primeira geração; * ASG: antipsicóticos segunda geração 
Os sinais e sintomas adversos mais importantes são apresentados de forma resumida na Tabela 5 e deverão ser cuidadosamente monitorizados em consultas de rotina pelo médico psiquiatra assistente (com eventual apoio do médico de família). Assim, análises sanguíneas e o eletrocardiograma (ECG) deverão ser realizados periodicamente, tal como medição de parâmetros vitais. ${ }^{4,6,31-33}$

\section{QUE FATORES DEVERÃO SER TIDOS EM CONTA NA ESCOLHA DA MEDICAÇÃO ANTIPSICÓTICA?}

Existem antipsicóticos disponíveis em formulações injetáveis utilizados em situações de urgência como em casos de agitação psicomotora. Os antipsicóticos injetáveis de longa ação (formulações depôt), com duração de semanas a meses, podem ter utilidade em doentes com baixa adesão à terapêutica oral. 4,6,31,32

A escolha do fármaco dependerá de vários fatores, entre os quais a clínica do quadro atual, os antecedentes pessoais do doente, tolerabilidade e as respostas prévias a outros antipsicóticos eventualmente realizados no passado. Os casos refratários à terapêutica antipsicótica podem beneficiar de electroconvulsivoterapia. Em qualquer dos casos, é importante salientar que a introdução de medicação antipsicótica provavelmente implica um encaminhamento para consulta com um médico especialista em psiquiatria. $4,6,31,32$

\section{QUAIS SÃO AS ABORDAGENS TERAPÊUTICAS NÃO FARMACOLÓGICAS E DE REABILITAÇÃO COGNITIVA?}

Relativamente ao tratamento não farmacológico, existem várias terapias psicológicas que podem desempenhar um papel importante na vida das pessoas com esquizofrenia. Alguns tratamentos específicos focados na adesão à terapêutica, na ansiedade social, no treino de competências sociais e na abordagem do uso de drogas têm eficácia comprovada nesta população. A criação de unidades de dia hospitalares, bem como associações na comunidade de apoio a esta população de doentes, contribuiu consideravelmente para o fortalecimento da sua reintegração social. $2,4,6,31,32$

\section{CONCLUSÃO}

A esquizofrenia é uma das doenças psiquiátricas mais comuns, mas também mais graves, e é vista como o ex-líbris da psiquiatria. Os conhecimentos acerca desta doen- ça multifatorial têm evoluído ao longo do tempo sendo que ainda hoje não é compreendida na sua totalidade. Os sintomas podem ser variados, o diagnóstico complexo, e a evolução heterogénea, o que origina muitas vezes, nos médicos não especialistas em psiquiatria, dúvidas e questões acerca desta doença limitante.

Embora existam tratamentos farmacológicos eficazes, o grande desafio na esquizofrenia reside não só na adesão dos doentes à medicação mas também na deteção precoce desta doença. Por outro lado, a medicação antipsicótica acarreta uma série de possíveis efeitos adversos que podem por si só limitar a qualidade de vida das pessoas com esquizofrenia. Este é um dos motivos que leva a que estes doentes padeçam de várias comorbilidades médicas e que naturalmente necessitem de frequentes cuidados de saúde. Assim, é quase certo que qualquer médico especialista irá ter contacto com doentes com esquizofrenia ao longo da sua carreira, sendo de especial importância o papel do especialista em medicina geral e familiar.

A complexidade no diagnóstico da esquizofrenia e no seu tratamento torna o acompanhamento especializado por um médico psiquiatra imprescindível. Nesse sentido, todos os clínicos deverão estar atentos para fatores de risco ou sintomas-chave desta doença e ter um papel ativo no encaminhamento adequado para consulta de psiquiatria, já que o prognóstico da doença é tanto melhor quanto mais cedo for o início do tratamento psicofarmacológico.

\section{PROTEÇÃO DE PESSOAS E ANIMAIS}

Os autores declaram que os procedimentos seguidos estavam de acordo com a Declaração de Helsínquia da Associação Médica Mundial.

\section{CONFIDENCIALIDADE DOS DADOS}

Os autores declaram ter seguido os protocolos do seu centro de trabalho acerca da publicação de dados.

\section{CONFLITO DE INTERESSES}

Os autores declaram que não há conflito de interesses na publicação deste artigo.

\section{FONTES DE FINANCIAMENTO}

Os autores declaram não ter recebido subsídios ou bolsas para a elaboração do artigo.

\section{REFERÊNCIAS}

1. Asher L, Fekadu A, Hanlon C. Global mental health and schizophrenia. Curr Opin Psychiatry. 2018;31:193-9.

2. Sadock BJ, Sadock VA, Ruiz P. Kaplan \& Sadock's synopsis of psychiatry: behavioral sciences/clinical psychiatry. Alphen aan den Rijn: Wolters Kluwer; 2014.

3. Castillejos MC, Martín-Pérez C, Moreno-Küstner B. A systematic review and meta-analysis of the incidence of psychotic disorders: the distribution of rates and the influence of gender, urbanicity, immigration and socio-economic level. Psychol Med. 2018;1-15.

4. Braz Saraiva C, Cerejeira D. Psiquiatria fundamental. Lisboa: LIDEL; 2014.

5. Kovács G, Almási T, Millier A, Toumi M, Horváth M, Kóczián K, et al. Direct healthcare cost of schizophrenia - European overview. Eur Psychiatry J Assoc Eur Psychiatr. 2018;48:79-92.

6. Figueira ML, Sampaio D, Afonso P. Manual de psiquiatria clínica. Lisboa: LIDEL; 2014

7. Björk Brämberg E, Torgerson J, Norman Kjellström A, Welin P, Rusner $M$. Access to primary and specialized somatic health care for persons with severe mental illness: a qualitative study of perceived barriers and facilitators in Swedish health care. BMC Fam Pract. 2018;19:12.

8. Garrabé J. História da esquizofrenia, um século para a compreender. Lisboa: Climepsi Editores; 2004. 
9. Silva RC da. Esquizofrenia: uma revisão. Psicol USP. 2006;17:263-85.

10. Feifel D, Shilling PD, MacDonald K. A review of oxytocin's effects on the positive, negative, and cognitive domains of schizophrenia. Biol Psychiatry. 2016;79:222-33.

11. Direção-Geral de Saúde. Programa Nacional de Intervenção em Saúde Infantil e Juvenil [Internet]. 2012. [consultado 2018 mai 06]. Disponível em: https://www.dgs.pt/documentos-em-discussao-publica/consultapublica-programa-nacional-de-saude-infantil-e-juvenil-jpg.aspx.

12. Dieguez S. Balzac's Louis Lambert: schizophrenia before Kraepelin and Bleuler. Front Neurol Neurosci. 2013;31:10-34.

13. Telles-Correia D. Capítulo II - Semiologia psicopatológica. In: TellesCorreia, editor. Manual de psicopatologia. $2^{\mathrm{a}}$ ed. Lisboa: LIDEL; 2014. p. 19-96.

14. Oyebode F. Sims' symptoms in the mind e-book: textbook of descriptive psychopathology. Philadelphia: Elsevier Health Sciences; 2014.

15. Fish FJ, Casey PR, Kelly B. Fish's clinical psychopathology: signs and symptoms in psychiatry. London: RCPsych Publications; 2007.

16. Lothane Z. The psychopathology of hallucinations-a methodological analysis. Br J Med Psychol. 1982;55:335-48.

17. Mitra S, Mahintamani T, Kavoor AR, Nizamie SH. Negative symptoms in schizophrenia. Ind Psychiatry J. 2016;25:135-44.

18. Associação Psiquiátrica Americana. Manual de diagnóstico e estatístico de perturbações mentais. $5^{\mathrm{a}}$ ed. Washington: APA; 2013

19. Organização Mundal de Saúde. Classificação Internacional de Doenças ICD-10. Genebra: OMS; 1994

20. Vaz-Serra A, Palha A, Figueira ML, Bessa-Peixoto A, Brissos S, Casquinha $P$, et al. Cognição, cognição social e funcionalidade na esquizofrenia. Acta Med Port. 2010;23:1043-58.

21. Krynicki CR, Upthegrove R, Deakin JF, Barnes TR. The relationship between negative symptoms and depression in schizophrenia: a systematic review. Acta Psychiatr Scand. 2018;137:380-90.

22. Upthegrove R, Marwaha S, Birchwood M. Depression and schizophrenia: cause, consequence, or trans-diagnostic issue? Schizophr Bull. 2017;43:240-4.

23. Jindal RD, Keshavan MS. Neurobiology of the early course of schizophrenia. Expert Rev Neurother. 2008;8:1093-100.

24. Madaan V, Bestha DP, Kolli VB. Biological markers in schizophrenia: an update. Drugs Today. 2010;46:661-9.

25. Associação Psiquiátrica Americana. Manual de diagnóstico e estatístico de perturbações mentais. $4^{\mathrm{a}}$ ed. Washington: APA; 2000.

26. Tandon R. The nosology of schizophrenia: toward DSM-5 and ICD-11. Psychiatr Clin North Am. 2012;35:557-69.

27. Sæther SG, Schou M, Kondziella D. What is the significance of onconeural antibodies for psychiatric symptomatology? A systematic review. BMC Psychiatry 2017;17:161.

28. Albus M. Clinical courses of schizophrenia. Pharmacopsychiatry. 2012;45:S31-5

29. van Os J, Kapur S. Schizophrenia. Lancet. 2009;374:635-45.

30. Afonso P. Esquizofrenia: para além dos mitos descobrir a doença. Cascais: Principia; 2010.

31. Taylor D, Paton C, Kerwin R. The Maudsley prescribing guidelines in psychiatry. 12 $2^{\text {th }}$ Edition. New Jersey: Wiley-Blackwell; 2015.

32. Castle DJ, Buckley PF. Schizophrenia. $2^{\text {nd }}$ ed. Oxford: Oxford University Press; 2015.

33. Stahl SM. Prescriber's guide: Stahl's essential psychopharmacology. $6^{\text {th }}$ Edition. Cambridge: Cambridge University Press; 2017.

34. Stahl SM. Stahl's essential psychopharmacology: neuroscientific basis and practical applications. $4^{\text {th }}$ ed. Cambridge: Cambridge University Press; 2013. 